\title{
Smart Home Hardware Design based on STM32
}

\author{
Hao Yin \\ Department of Electrical Engineering, North China Electric Power University (Baoding), Baoding \\ 071000, China; \\ Email:jsyz165@gmail.com
}

Keywords: Smart home, STM32, sensor, IOT.

\begin{abstract}
This paper discuss the part of temperature, humidity and light intensity sensor in the smart home system based on the STM32. The system uses the DHT11 digital temperature and humidity sensor to get and send the data, and uses the BH1750FVI to perceive light intensity. The paper also discuss the transmission timing and the data format of each sensor. At the end of the paper, it is given the $\mathrm{C}$ code in the KEIL MDK environment.
\end{abstract}

\section{Introduction}

The world's first intelligent building appears in the Connecticut State in 1984, which was only a traditional building with a certain renovation. The building just used the computer to detect and control the elevator, lighting, air condition and other equipment. This building marks the prelude to the smart home. [1]

Nowadays, with the development of the microchip technology,chips of low power cost have become more and more cheap.Therefore,the system based on the STM32 or other chips makes it easier to get aware of the operating status of electricity at anywhere and anytime, which can improving the convenience of people's daily lives and safety.

\section{Hardware department}

The main hardware of the design are the following items, and there are short instructions.

\section{STM32F103}

This project chosen STM32F103 [2] belongs to the enhanced model in the series of STM32.The clock frequency of the enhanced series can be multiplied to $72 \mathrm{MHz}$, which is the high speed product in the similar products .STM32F103 has a rich interface and not a small built-in memory.

STM32F103 is a chip produced by ST Company based on the Cortex-M3 kernel, which has a rich interface and not a small built-in memory. Because the chip has an internal flash, there is no need to configuring external ROM, which can simplify the system. When the clock frequency to achieve maximum $72 \mathrm{MHz}$, the normal operating current is $36 \mathrm{~mA}$.

The STM32F103 has three modes [3]: Sleep Mode, Stop Mode, and Standby Mode. In Sleep mode, only the CPU is stopped. All peripherals continue to operate and can wake up the CPU when an interrupt/event occurs. The Stop mode achieves the lowest power consumption while retaining the content of SRAM and registers. All clocks in the $1.8 \mathrm{~V}$ domain are stopped, the PLL, the HSI RC and the HSE crystal oscillators are disabled The Standby mode is used to achieve the lowest power consumption. The internal voltage regulator is switched off so that the entire $1.8 \mathrm{~V}$ domain is powered off.

\section{DHT11}

DHT11 [4] digital temperature and humidity sensor is one that contains a calibrated digital signal output from the temperature and humidity sensor complex. Accuracy: Humidity $\pm 5 \% \mathrm{RH}$, temperature $\pm 2^{\circ} \mathrm{C}$, humidity range of $20 \sim 90 \% \mathrm{RH}$, temperature $0 \sim 50{ }^{\circ} \mathrm{C}$.

DHT11 digital temperature and humidity sensor applications dedicated digital modules and acquisition of temperature and humidity sensing technology. Its interior consists of a resistor and a sense of wet NTC temperature measurement components, higher progress, can be used to improve the 
reliability and excellent long-term stability testing, and with a high-performance 32-bit microcontroller connected. Thus, the product has excellent quality, fast response, anti-interference ability, high cost performance.

\section{BH1750FVI}

This project uses a 16 digital light intensity sensor BH1750FVI released by ROHM Co., Ltd. It is a chip having a standard communication interface of IIC, which has the feature of weak light dependence, anti-interference ability.

Traditional light intensity sensor mainly uses photosensitive resistance, optical characteristics and its working principle is: photo resistor current

The relationship between the illuminance. However, due to the photoelectric characteristics of the photo resistor not ideal linear relationship, so it was not used as a high-precision detection element.

In addition, the photo resistor is not easily visible (mainly infrared and ultraviolet) impact caused by misuse. And BH1750FVI This is a light intensity sensor, high accuracy, short measuring time and the circuit design is relatively simple, relatively easy to implement and integrate, the current has been widely used in various types of embedded systems, mainly for mobile phones, LCD TV, lighting control, digital cameras, car navigation systems, etc. [5]

\section{Software Development Environment}

Keil MDK is the most comprehensive software development solution for ARM Cortex-M microcontrollers. It includes IDE, C/C++ compiler, debugger, Software Pack management, and CMSIS.

\section{Smart home hardware system implementation}

\section{DHT11 data format}

DHT11 uses single bus data format and .follows the high first-out rule. The whole data is 40-bit, including 16-bit temperature data, 16-bit humidity data and 8-bit checksum data. Each 16-bit data has 8-bit integer and 8-bit decimal. Supposing the transmission is correct, the checksum data should be equal to last eight bit of the summary by adding the previous four 8-bit data. For example, the humidity is $39.80 \%$ and the temperature is $21.50^{\circ} \mathrm{C}$, the corresponding 40 -bit data is 00100111,0101 0000, 0001 0101, 0011 0010, 1011, 1110.

The following sheet maybe more direct.

\begin{tabular}{lccc}
\hline & Bin & Hex & Dec \\
\hline Humidity integer & 00100111 & $27 \mathrm{H}$ & 39 \\
Humidity decimal & 01010000 & $50 \mathrm{H}$ & 80 \\
Temperature integer & 00010101 & $15 \mathrm{H}$ & 21 \\
Temperature decimal & 00110010 & $32 \mathrm{H}$ & 50 \\
Checksum & 10111110 & BEH & \\
\hline
\end{tabular}

\section{DHT11 Timing}

The communication process is shown in the following picture, (the solid line is the host signal, and the dotted line is the DHT11 signal).First, the STM32F103 host will send a start signal. When the DHT11 detected the start signal, it will give a response output and give the high level to the data line .This response means the sensor will send the measured temperature data and humidity data to the host .In the end, the bus line will be released after the sending. 


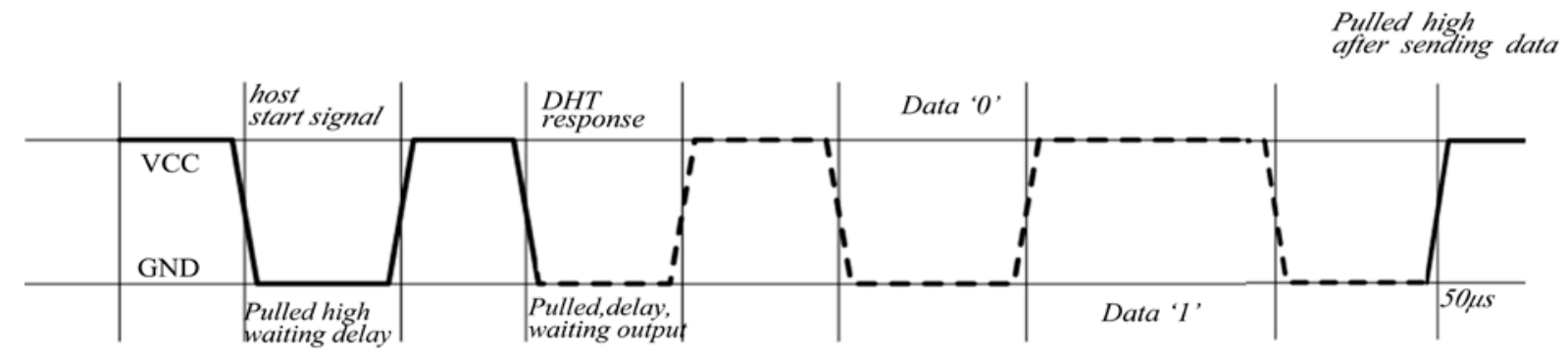

\section{Example code of DHT11}

Fig.1 DHT11 transmission timing

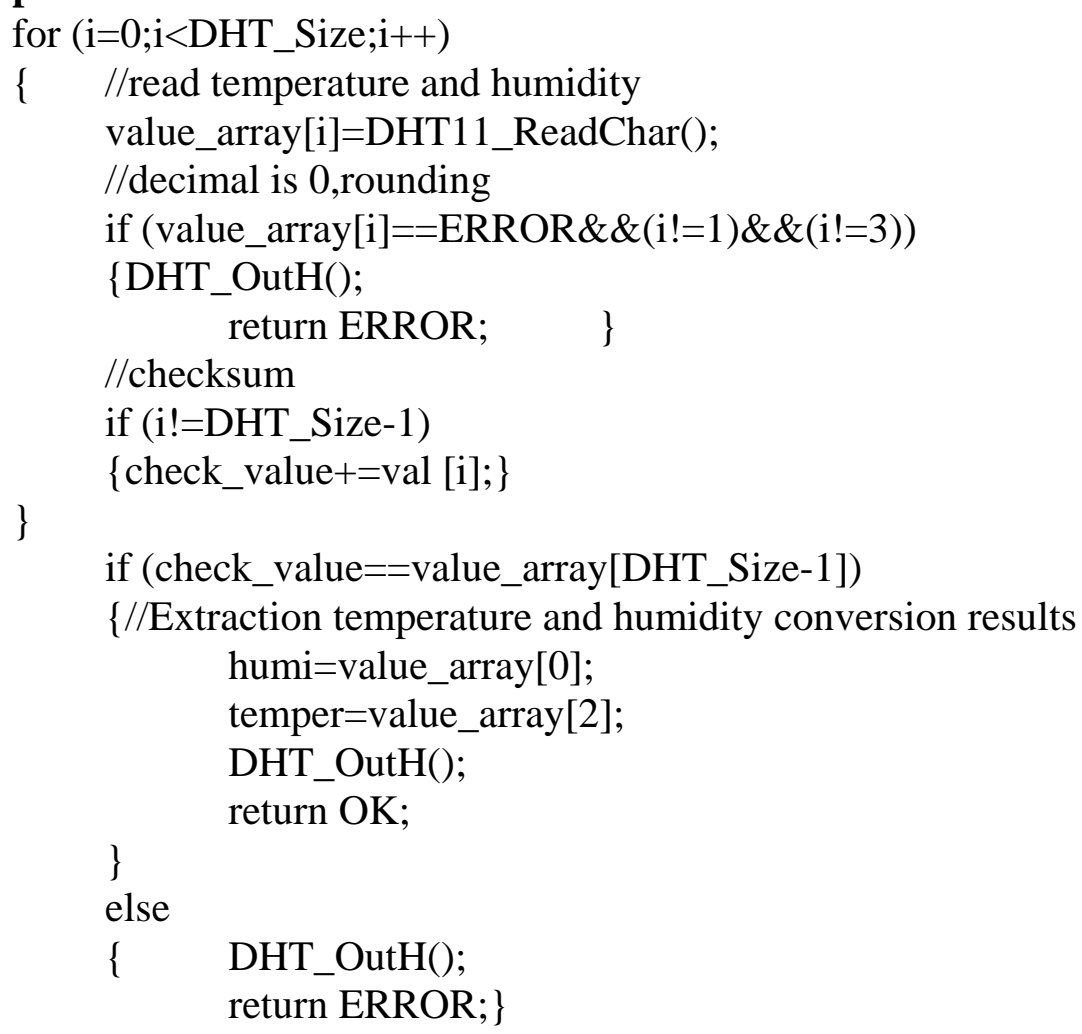

\section{BH1750FVI data format}

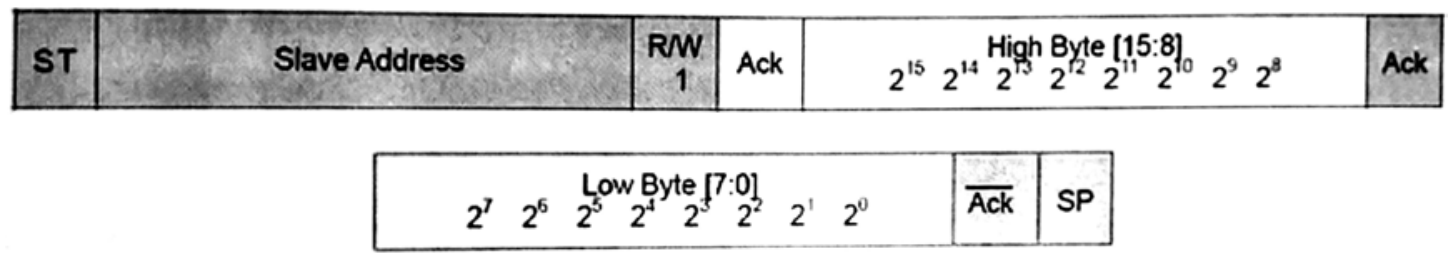

Fig.2 BH1750FVI data format

There gives an example to illustrate:

Assuming the High Byte $=10000011$, Low Byte $=10010000$

Then the real result is

$\left(2^{15}+2^{9}+2^{8}+2^{7}+2^{4}\right) / 1.2=28067 \mathrm{~lx}$

\section{BH1750FVI timing}

Fig. 3 shows the transmission timing of BH1750FVI, which is more complex than the timing of the DHT11. 


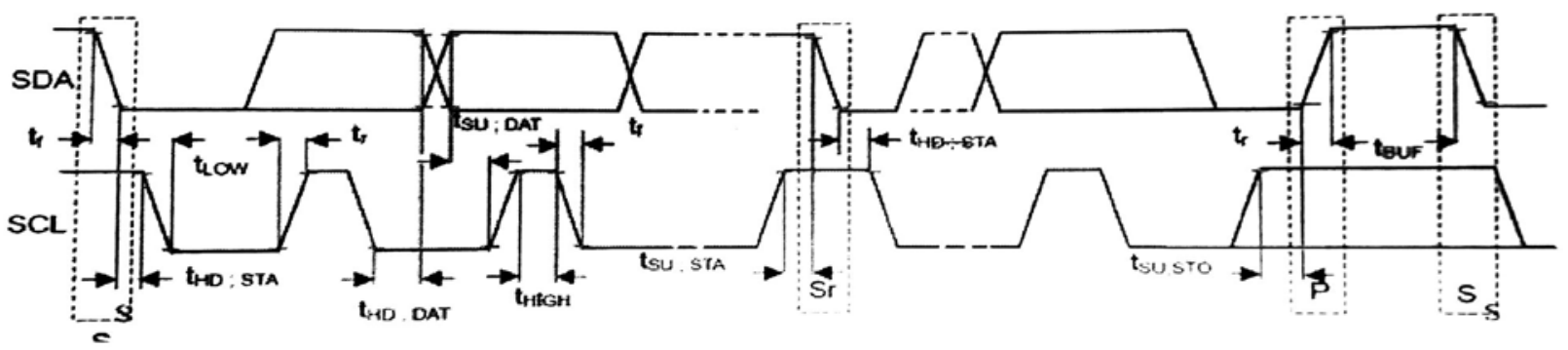

\section{Example code of BH1750FVI}

float BH1750_ReadResult()

\{

u8 result $\mathrm{H}=0$,result $\mathrm{L}=0$;

float result $=0$;

//start signal

BH1750_Start();

//send device address and WR signal

BH1750_SendByte(SlaveAddress+1);

//read high data

resultH=BH1750_ReadByte();

BH1750_SendACK(0);

//read low data

resultL=BH1750_ReadByte();

BH1750_SendACK(1);

result=(resultH*pow $(2,8)+$ resultL $) / 1.2$;

//send stop signal

BH1750_Stop();

return result;

\}

\section{Conclusion}

The system applies the single bus sensor and uses the STM32 chip to achieve the detection of temperature and light intensity. With the additional relay control and switching control, the automatically control under different conditions can be implemented.

\section{References}

[1] Wacks K. Home systems standards: achievements and challenges[J]. Communications Magazine, IEEE, 2002, 40(4): 152-159.

[2] SUN S, CHEN Z, KOU C. The Developing and Application of the New Generation of Embedded MPU-STM32F103 [J][J]. Microcomputer Applications, 2010, 12: 009.

[3] Available from Internet :

http://www2.st.com/content/ccc/resource/technical/document/datasheet/33/d4/6f/1d/df/0b/4c/6d/CD 00161566.pdf/files/CD00161566.pdf/jcr:content/translations/en.CD00161566.pdf

[4] The module product manual of temperature and humidity: DHT11[EB/OL]. http://www.aosong.com

[5] Zhang Y M, Fu S J. Stm 32's Controling System of Intelligent Greenhouse[J]. RuanjianSoftware, 2010, 31(12): 14-18. 